\title{
Nasendoscopic Findings of Velopharyngeal Sphincter in Operated Cleft Palate Patients: Is It Different than Normal Population
}

\author{
Akangsha Sharma ${ }^{1}$ Shamendra Anand Sahu ${ }^{2}$ Karoon Agrawal ${ }^{3}$ \\ ${ }^{1}$ Department of Plastic and Cosmetic Surgery, BLK Super Speciality \\ Hospital, New Delhi, India \\ ${ }^{2}$ Department of Plastic, Reconstructive and Burn Surgery, All India \\ Institute of Medical Sciences, New Delhi, India \\ ${ }^{3}$ Department of Burns, Plastic and Maxillofacial Surgery, Safdarjung \\ Hospital and VMMC, New Delhi, India \\ Indian J Plast Surg 2019;52:178-182
}

\begin{abstract}
Address for correspondence Karoon Agrawal, MBBS, MS, MCh, E 44, Second Floor, Panchsheel Park, New Delhi 110017, India (e-mail: karoonaparna@gmail.com).
\end{abstract}

\author{
Abstract \\ Keywords \\ - velopharyngeal \\ closure \\ - velopharyngeal port \\ - nasendoscopy \\ - cleft palate \\ - velopharyngeal \\ incompetence \\ - velopharyngeal \\ sphincter \\ - palatoplasty
}

Objective This study was aimed for nasendoscopic assessment of velopharyngeal sphinteric closure in patients with operated cleft palate and to compare it with normal population.

Design A cross-sectional study was done in a tertiary cleft care center in 30 patients with operated cleft palate after a minimum of 6 months of their surgery and 30 randomly selected volunteers with normal speech. Both groups were one-time evaluated by three observers using 70 degree rigid nasendoscope and/or pediatric fiber optic endoscope. Velopharyngeal sphincter closure characteristics in terms of pattern of closure, dominant element involved in the closure, degree of palatal movement, and completeness of the closure were evaluated, recorded, and compared between the groups.

Results In both groups, the most common pattern of closure is coronal and soft palate is the dominant mobile element in velopharyngeal closure. All normal subjects showed complete closure of the sphincter with good soft palate movement. But only $50 \%$ of the operated patients with cleft showed complete closure and even less than them had good movements of the soft palate.

Conclusions Although the pattern of the closure in the operated patients is similar to the normal subjects, the movement of the soft palate and completeness of the velopharyngeal sphincter closure still remain the problem in the operated palate patients.

\section{Introduction}

Velopharynx is defined as space connecting nasal and oral cavities. Respiration, swallowing, and speech production are important functions which require separation of these two anatomical cavities by closure of the velopharyngeal space. Anatomically the velopharyngeal space is surrounded by velum anteriorly, pharyngeal walls on both sides, and posteriorly. ${ }^{1}$ The velopharyngeal port with surrounding muscular structures form a dynamic three-dimensional (3D) muscular valve referred to as "velopharyngeal sphincter." ${ }^{2}$ The contraction of various muscular components closes the port during speech production.

The closure pattern of the velopharyngeal port varies among individuals, though closure of the port varies with different speech sounds. The plosives require complete velopharyngeal closure, while vowels and nasal consonants require variable opening of velopharyngeal port. ${ }^{2}$ There are four established closure pattern of velopharyngeal valve, namely, coronal, circular, sagittal, and circular with Passavant's ridge. ${ }^{3}$ The last pattern is also called as "bowtie pattern" (-Video 1). ${ }^{4}$
DOI https://doi.org/

$10.1055 / \mathrm{s}-0039-1696634$

ISSN 0970-0358.
License terms

(이 (1) $\Theta \circledast$ 


\section{Video 1}

Nasendoscopy showing circular pattern with Passavant's ridge. Online content including video sequences viewable at: www.thieme-connect.com/products/ejournals/ html/10.1055/s-0039-1696634.

Perceptual speech assessment, nasendoscopy, and video fluoroscopy are now commonly performed to evaluate pre and postoperative results of palatoplasty. Sagar and Nimkin have now reported pre and postoperative 3D magnetic resonance imaging (MRI) assessment of airway during speech for velopharyngeal surgery. ${ }^{5}$

There is always a doubt that does the cleft palate or repair of cleft palate affect the natural mobility of the velopharyngeal sphincter. With this question in mind, the nasendoscopic findings in operated patients of cleft palate were compared with those of normal volunteers.

\section{Methods}

This is a cross-sectional study done from December 2013 to June 2015 in a tertiary cleft care hospital to assess the nasendoscopic findings in patients with operated cleft and compare the velopharyngeal sphinteric closure with the cohort having normal speech. This study was cleared by the protocol committee and institute ethical committee. Two study groups of 30 patients each between 10 and 30 years were formed. The older age group was selected as nasendoscopy is feasible in awake patients under local anesthesia and they can easily follow commands.

In control group, patients with normal speech were included. In other group, operated cleft patients after 6 months of surgery were included. The patients were classified as per Nagpur classification advocated by Prof. Chakkiri Balakrishnan. ${ }^{6}$ Most were cleft group-II patients. Few patients were in group III, already operated for lip. Veau-Wardill-Kilner palatoplasty or Bardach's palatoplasty was done according to the length of palate. Patients who developed postoperative palatal fistula were excluded from the study.

After counseling and appraisal, written informed consent was obtained from each patient included in the study. Prior to nasendoscopy, the nasal cavity of the patients were anaesthetized locally by spraying $10 \%$ lignocaine and applying $4 \%$ lignocaine viscous using the swab sticks inserted into the nostrils. Both the groups of the patients were evaluated with the 70-degree rigid endoscope and/or pediatric fiber optic nasendoscope available in the department by three observers. The larger and patent nostril was chosen. The endoscope was passed along the inferior or middle meatus of the nasal cavity to view the velopharyngeal portal. To study the dynamic sphincter, the subjects of both the groups were instructed to count 1 to 10 and from 60 to 70 and were asked to speak certain sets of words. The speech and endoscopic video (using Fentex Hi cam Nasendoscopy System) were recorded simultaneously using computer software (Cyberlink power director). The recorded video was repeatedly played for three observers. Two observers were internal and one observer was external practicing plastic surgery. The observations of the three observers are recorded separately and most common of the three observations was taken. Speech evaluation was not done because it was beyond the scope of the study. Observations were made in both groups as per the points shown in the - Table 1 .

\section{Results}

In our study, we categorized the patients in age groups to see if the patients are statistically comparable as shown in

\section{-Fig. 1.}

On applying Chi-square test, there was no significant difference $(p$-value $=0.76)$ between two groups. In our study, there were 19 males and 11 females out of 30 in the normal population group and 24 males and 6 females out of 30 in the postoperative group ( $p$ value is not significant i.e. 0.15 ).

Using nasendoscopy examination, we are able to visualize the dominant mobile element closing the velopharyngeal port in both the study groups as shown in - Fig. 2. On

Table 1 Velopharyngeal function evaluation done for the following parameters

\begin{tabular}{|l|l|}
\hline 1. & $\begin{array}{l}\text { The pattern of velopharyngeal closure } \\
\text { A. Circular } \\
\text { B. Coronal } \\
\text { C. Circular with Passavant's ridge } \\
\text { D. Sagittal } \\
\text { E. Irregular }\end{array}$ \\
\hline 2. & $\begin{array}{l}\text { Degree of palatal movement while velopharyngeal } \\
\text { closure } \\
\text { A. Good } \\
\text { B. Moderate } \\
\text { C. Poor }\end{array}$ \\
\hline 3. & $\begin{array}{l}\text { Dominant mobile element in the velopharyngeal } \\
\text { sphincter closure } \\
\text { A. Soft palate } \\
\text { B. Lateral pharyngeal wall } \\
\text { C. Posterior pharyngeal wall }\end{array}$ \\
\hline 4. & $\begin{array}{l}\text { Degree of closure of velopharyngeal sphincter } \\
\text { A. Complete } \\
\text { B. Incomplete/inadequate } \\
\text { C. Wide open }\end{array}$ \\
\hline
\end{tabular}

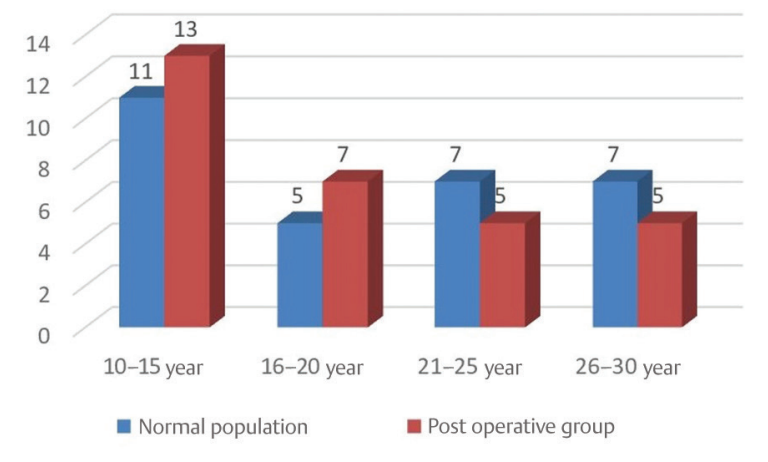

Fig. 1 Distribution of patients according to age. 


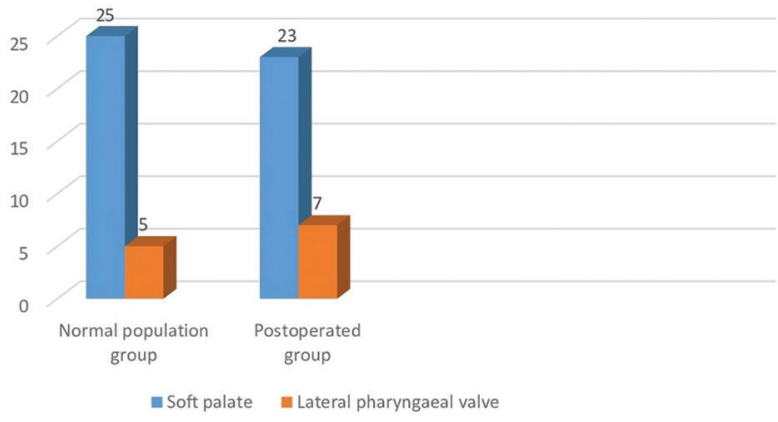

Fig. 2 Figure showing dominant element in closure of velopharyngeal port.

Table 2 Distribution of patients according to pattern of velopharyngeal closure

\begin{tabular}{|l|l|l|}
\hline Pattern & $\begin{array}{l}\text { Normal } \\
\text { population } \\
(\boldsymbol{n}=30)\end{array}$ & $\begin{array}{l}\text { Postoperative } \\
\text { group }(\boldsymbol{n}=30)\end{array}$ \\
\hline Circular & $8(\%)$ & 7 \\
\hline Coronal & 10 & 17 \\
\hline $\begin{array}{l}\text { Circular with Passavant's } \\
\text { ridge }\end{array}$ & 8 & 2 \\
\hline Sagittal & 2 & 2 \\
\hline Irregular & 2 & 2 \\
\hline
\end{tabular}

applying the Chi-square test, there was no significant difference $(p$-value $=0.14)$ between the two groups.

The pattern of closure of the velopharyngeal closure in both the groups has been summarized in - Table 2 . Chi-square test showed no significant difference $(p=0.14)$ between the two groups.

All the normal patients showed good palatal movement and have complete closure of the velopharyngeal port. But in postoperative group, only 12 patients showed complete closure of the port, whereas nine had wide open port and rest of the nine had incomplete closure. While assessing the palatal movement, there were 15 individuals with good palatal movements, but seven were classified as moderate and rest of the eight individuals had poor palatal movement.

\section{Discussion}

Patients with cleft palate have difficulty in speech and articulation. This results from abnormal anatomy of the soft palate musculature causing inadequate functioning of the velopharyngeal port. Pre- and postoperative assessment of the velopharyngeal port and sphincter mechanism is a prerequisite in cleft palate patients to match the outcome of an intervention for optimum speech outcome. Simple perceptual speech evaluation provides easy identification of impaired velopharyngeal sphinter but tells nothing about the anatomy and closure characteristics that underlie the impairment. Assessment of velopharyngeal sphincter can be performed by direct visualization using nasendoscopy and or fluoroscopy and the effect of the sphincter on physical parameters like sound, airflow, and air pressure. ${ }^{2}$ All the above techniques have some merits and limitations. The direct visualization of the sphinteric mechanism using nasendoscopy is cheap, easy, and reproducible method of evaluation in pre- and postoperative period. The present study was undertaken to evaluate the characteristics of velopharyngeal sphincter closure in operated patients of cleft palate and compare with that of normal population, using nasendoscopy.

Croft et al in 1981 found multiple patterns of velopharyngeal valving in 80 normal and 500 operated patients of cleft palate using nasopharyngoscopy and video fluoroscopy. Authors described coronal, sagittal, circular, and circular with Passavant's ridge patterns of closure in both the groups and concluded that the coronal is the most common pattern of closure in both the groups. ${ }^{2}$ Also, different patterns of closure were found in similar frequency in both normal and postoperated population. In our study, coronal type of velopharyngeal valve closure was most common finding ( - Video 2). Frequency of occurrence of other various patterns of closure (circular, sagittal, circular with Passavant's ridge, and irregular) in both the groups showed no significant difference ( $p=0.14 ;-$ Fig. 3 ). This indicates that cleft palate and its repair do not change the velopharyngeal closure pattern in a given population. But in a study by Manochiopining et al on normal Thai individuals, circular was the most common pattern of closure (-Video 3). ${ }^{7}$ The assessment of pattern of closure is a subjective observation. It is likely that different observers have difference in the assessment.

\section{Video 2}

Nasendoscopy showing coronal type of closure pattern. Online content including video sequences viewable at: www.thieme-connect.com/products/ ejournals/html/10.1055/s-0039-1696634.
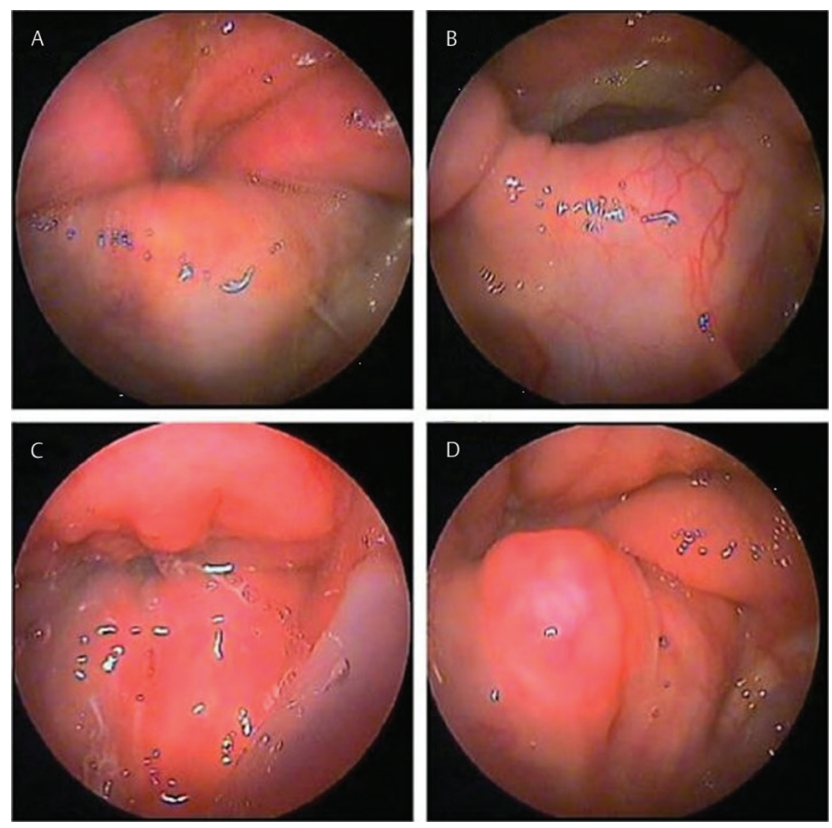

Fig. 3 Different types of closure pattern; (A) circular type, (B) circular with Passavant's ridge, (C) coronal type, and (D) irregular type. 


\section{Video 3}

Nasendoscopy showing circular type of closure pattern. Online content including video sequences viewable at: www.thieme-connect.com/products/ ejournals/html/10.1055/s-0039-1696634.

Normal population have good palatal movement in all the individuals with complete closure of the velopharyngeal port as in our study, where as in postoperative group only 15 out of 30 individuals had good palatal movements. On searching the literature, we could not find study evaluating the movement of palate postoperatively. Although Igawa et al in 1998 described three types of velopharyngeal closure patterns in cleft palate patients postoperatively, depending on the dominant element closing the velopharyngeal sphincter, namely, (1) soft palate type, in which the soft palate mainly operates; (2) lateral wall type, in which compensational medial movement of the lateral pharyngeal wall is mainly observed; and (3) mixed type, in which both the soft palate and the lateral pharyngeal walls operate. ${ }^{8}$

Good palatal movement cannot be the sine qua non of the complete closure of the velopharyngeal sphincter. Of our 30 patients of postoperative group, despite good soft palate movements in 15 , only 12 patients showed complete closure of the velopharyngeal port on the nasendoscopy. Matsuya et al also reported that his 40 of 68 patients did not achieve complete closure during any activity operated for cleft palate. ${ }^{9}$ Similarly, Yellinedi et al $^{10}$ performed videofluroscopy in 117 operated cleft palate patients and reported 48 patients had a resting gap of $>10 \mathrm{~mm}$ who did not achieve velopharyngeal (VP) closure on phonation, thus having full blown velopharyngeal incompetence (VPI). In our study, nasendoscope has been used that permits subjective analysis regarding closure of sphincter. There is no objectivity as we cannot measure the exact gap size in incomplete closure.

The determination of the dominant element in the valving mechanism is important for planning surgery for velopharyngeal incompetence. In our both study groups, the dominant element of velopharyngeal valving is soft palate. Various sphincter pharyngoplasty procedures bring the tissue toward the center and thus is most useful for closure pattern with lateral defects like coronal and bowtie patterns in which lateral wall motion is poor. Similarly, after pharyngeal flap surgery, the closure of the lateral pharyngeal port requires lateral wall motion. Hence, it can only be effective in patients with sagittal or circular closure patterns having adequate lateral wall motion. ${ }^{4}$

From the above results and discussion, it is clear that there is no significant difference between the mobile dominant element and closure patterns of normal population versus postoperative individuals of cleft palate indicating that velopharyngeal valving is not physiologically affected by surgery. Although due to small sample size of the study groups there

\section{Video 4}

Nasendoscopy showing Irregular type of closure pattern. Online content including video sequences viewable at: www.thieme-connect.com/products/ ejournals/html/10.1055/s-0039-1696634.

are chances of type-II statistical error. Apart from four established closure pattern of velopharyngeal valve, namely, coronal, circular, sagittal, and circular with Passavant's ridge, ${ }^{3}$ we have described a new "irregular type" closure pattern after examining the normal and operated patients of cleft palate patients in our study (-Video 4). The degree of palatal movement and thus completeness of the closure in postoperative patient depends upon multiple factors like degree of clefting and meticulous surgical technique. Nasendoscopy plays a pivotal role in the evaluation of operated cleft palate patients and planning surgical management velopharyngeal insufficiency associated with cleft palate repair. Surgery may be avoided if nasendoscopy does not reveal a significant defect and an appropriate speech therapy can be assigned to the patient; later surgery may be planned in case of indication after adequate speech therapy and evaluation.

Despite so many advantages, nasendoscopy does have its shortcomings. Information obtained to assess the velopharyngeal port is in the ratio or percentage rather than an absolute value. It is difficult to obtain standardized views or infer relative dimensions. Patient cooperation is a critical factor that further limits the use of nasendoscopy, especially in pediatric population. The examination relies on the observer for interpretation of results, making it a subjective investigation.

\section{Disclosures}

- All authors have no financial disclosure to make.

- None of the authors has a financial interest in any of the products, devices, or drugs mentioned in this manuscript.

- The manuscript, figures, tables, and data are not been published previously and are not under consideration for publication elsewhere.

\section{Conflict of Interest Statement}

All the authors have no conflict of interest to declare.

\section{References}

1 Perry JL. Anatomy and physiology of the velopharyngeal mechanism. Semin Speech Lang 2011;32(2):83-92

2 Marsh JL. Velo-pharyngeal dysfunction: evaluation and management. Indian J Plast Surg 2009;42(Suppl):S129-S136

3 Croft CB, Shprintzen RJ, Rakoff SJ. Patterns of velopharyngeal valving in normal and cleft palate subjects: a multi-view videofluoroscopic and nasendoscopic study. Laryngoscope 1981;91(2):265-271

4 Woo AS. Velopharyngeal dysfunction. Semin Plast Surg 2012;26(4):170-177 
5 Sagar P, Nimkin K. Feasibility study to assess clinical applications of 3-T cine MRI coupled with synchronous audio recording during speech in evaluation of velopharyngeal insufficiency in children. PediatrRadio 2015;45(2):217-227

6 Subramani SA, Murthy BS. A classification of cranio facio cervical (branchial) clefts (Bangalore classification). Indian J PlastSurg 2005;38(2):79-94

7 Manochiopining S, Chuangsuwanich A, Taweepraditpol S. The nasendoscopic study of velopharyngeal closure patterns in normal thai volunteers. Siriraj Med J 2005;5:118-121
8 Igawa HH, Nishizawa N, Sugihara T, Inuyama Y. A fiberscopic analysis of velopharyngeal movement before and after primary palatoplasty in cleft palate infants. Plast Reconstr Surg 1998;102(3):668-674

9 Matsuya T, Yamaoka M, Miyasaki T. A fiberscopic study of velopharyngeal closure in patients with operated cleft palates. Plast Reconstr Surg 1979;63(4):497-500

10 Yellinedi R, Damalacheruvu MR. Is there an optimal resting velopharyngeal gap in operated cleft palate patients? Indian J Plast Surg 2013;46(1):87-91 